\title{
On Professional Standards of Publication in Peer-Reviewed Research Journals
}

Hojjat Adeli, Hon. M., ASCE, Editor-in-Chief, Computer Aided Civil and Infrastructure Engineering, Abba G. Lichtenstein Professor, The Ohio State University, 470 Hitchcock Hall, 2070 Neil Avenue, Columbus, OH 43210

William Rasdorf, F. ASCE, Editor, Journal of Computing in Civil Engineering, Professor, Department of Civil, Construction, and Environmental Engineering, North Carolina State University, Campus Box 7908, Raleigh, NC 27695

An important premise of rigorously peer-reviewed research journals is that each paper must be the author's original and unpublished work. Authors can present a preliminary version of their paper at a conference to receive feedback from fellow researchers to improve their research. However if that paper is subsequently submitted to a peer-reviewed research journal it must be significantly different from the preliminary version published in the conference proceedings.

Simultaneous submission of a paper to two different journals is universally accepted to be a breach of professional scholarly conduct. Journal editors have to grapple with the issue of simultaneous submission with great uncertainty. The purpose of this editorial is to bring this issue to light and perhaps warn the potential violators that the journal editors are watching! One clue to simultaneous submission is when an author abruptly withdraws his/her paper from a journal. Some authors submit minor variations of the same paper to two different journals. Presented below are 3 examples of such violations.

\section{Cases and Consequences}

A technical note published in Micocomputers in Civil Engineering (the former title of ComputerAided Civil and Infrastructure Engineering during 1986-1997), 10:2, March 1995, was republished in a substantially similar form in the October 1995 issue of ASCE Journal of Computing in Civil Engineering (JCCE). A year later, a paper published in Microcomputers in Civil Engineering, 11:2, March 1996, was republished in the April 1998 issue of Journal of Computing in Civil Engineering in a substantially similar form. In both cases these papers eluded the checks and balances of the review process. However, upon subsequent discovery, these authors were banned from publishing for a number of years.

These previous two violations were made some ten years ago. But recently there has been another serious violation which prompted us to write this editorial. Last year, two companion papers were submitted for possible publication in Computer-Aided Civil and Infrastructure Engineering (CACAIE). The authors were from a major research university in the U.S. and another research university overseas. A reviewer of CACAIE, who happened to be an associate editor of JCCE, noted that a significant part of these papers had been included in another paper submitted to JCCE which was already accepted for publication in JCCE and was in queue to be published. The selection of that particular reviewer by the editor of CACAIE was in fact made deliberately and vigilantly as a matter of his editorial style. 
While the previous two cases were recognized after the act, this one was discovered during the violation. After identifying the duplicate submission the authors were notified. They subsequently submitted letters of apology. The outcome of the situation was that the reviews of the papers submitted to CACAIE were suspended. The authors also received disciplinary action by ASCE and the JCCE. First, the decision to publish the paper was reversed and it was not published. Second, there will be a period of years during which the JCCE will not publish papers from those authors.

\section{Procedures for Monitoring set by CACAIE}

Contributors to CACAIE (and the JCCE) are asked to provide a statement that the submitted manuscript is their original unpublished work and the manuscript (or any variation of it) has not been submitted to another publication previously. If the manuscript is an extension of the authors' previously published paper the editor of CACAE asks the authors to send him that paper also so that the reviewers can determine the archival significance and scientific originality of the current submission compared with their previous publication. For a paper published in the proceedings of a conference to be considered for possible publication in CACAIE (or in the JCCE) it has to be extended by additional original computational materials in a substantive way. In that case the authors are asked to forward a copy of their published conference paper which will be forwarded to reviewers to determine the level of expansion.

Further, when a graduate student, post doctoral researcher, or a junior researcher is the co-author of a manuscript the editor of CACAE asks the professor/advisor/senior researcher to submit it and serve as the corresponding author. This is done because he/she is in an experienced position and can better discern issues of originality, copyright assignment, and the high standard of publication of rigorously peer-reviewed research journals. This policy is based on experiencing numerous poorly prepared submissions and ethical violations by graduate students or junior researchers where in most cases the senior researcher or professor claimed subsequently that his co-author had submitted the paper without his knowledge!

\section{Responsible Parties}

Those who have an opportunity to identify ethical violations in the publishing arena once they have occurred include editors, reviewers, and the technical community. Editors can implement procedures like those identified above. Reviewers can diligently and carefully assess a paper and be aware of their role as a watchdog as well as a reviewer. Both editors and reviewers have the potential to identify ethical breaches before they are published, as was the situation in the third case above.

Those who have an opportunity to prevent ethical violations are authors. It is their duty to think carefully about the content of each paper so that it does not contain a large portion of the content of another paper. It is their duty to ensure that each paper they write has a substantive contribution to the technical community that is new and original. 